\title{
A numerical study of the SPH method for simulating transient viscoelastic free surface flows
}

\author{
Jiannong Fang ${ }^{1}$ \\ Laboratory of Engineering and Environmental Geology (GEOLEP), Institute of \\ infrastructures, resources and environment, Swiss Federal Institute of Technology \\ Lausanne, EPFL-ICARE, CH-1015 Lausanne, Switzerland \\ Robert G. Owens \\ Département de mathématiques et de statistique, Université de Montréal, CP 6128 \\ succ. Centre-Ville, Montréal QC H3C 3J\%, Canada \\ Laurent Tacher, Aurèle Parriaux \\ Laboratory of Engineering and Environmental Geology (GEOLEP), Institute of \\ infrastructures, resources and environment, Swiss Federal Institute of Technology \\ Lausanne, EPFL-ICARE, CH-1015 Lausanne, Switzerland
}

\begin{abstract}
The smoothed particle hydrodynamics (SPH) method is extended and tested for the numerical simulation of transient viscoelastic free surface flows. The basic equations governing the free surface flow of an Oldroyd-B fluid are considered and approximated by SPH. In particular, a drop of an Oldroyd-B fluid impacting a rigid plate is simulated. Results for a Newtonian fluid are also presented for comparison. It is found that the original SPH method, which has been successfully applied to the simulation of transient viscoelastic flows in bounded domains (such as the start-up flow between parallel plates), is unable to simulate the viscoelastic free surface flow considered here because of the so-called tensile instability. This instability leads to unrealistic fracture and particle clustering in fluid stretching and may eventually result in complete blowup of the simulation. Recent works have shown that in simulations of elastic solids the tensile instability can be removed by an artificial stress. Here we show that the same idea also works for viscoelastic fluids provided that the constant parameter entering in the definition of the artificial stress is properly chosen. Numerical results obtained are in good agreement with those simulated by a finite difference technique.
\end{abstract}

Key words: Smoothed Particle Hydrodynamics; Free surface; Viscoelastic flow; Oldroyd-B; Tensile instability 


\section{Introduction}

Simulation of free surface flows is a very important and active research area in computational fluid dynamics. Traditionally, grid-based numerical methods such as finite difference methods (FDM), finite volume methods and finite element methods (FEM) are used to simulate free surface flows. However, dealing with moving free surfaces and large deformations is generally a difficult task for the grid-based methods just mentioned. Over the last few decades, the main focus has been on capturing the free surface and regenerating the grid. The methods proposed include PIC (particle-in-cell) [1], MAC (markerand-cell) [2], VOF (volume-of-fluid) [3] and LS (level-set) [4] methods. The PIC and MAC methods use particles (markers) to locate the moving surfaces and have been widely used to solve complex free surface problems. Despite being flexible and robust, PIC and MAC are quite complicated in programming and expensive in computation especially for three-dimensional cases. The VOF method is inherently linked to fluid mechanics problems by solving an additional partial differential equation for the filled fraction of each control volume. The LS method employs an additional implicit level-set function to describe the advection of the moving surface. The equation for the level-set function is commonly solved in an Eulerian framework by using high order finite difference methods.

In the non-Newtonian fluid mechanics community, viscoelastic free surface flows have been studied for more than 30 years. Tanner [5] in 1970 made an early attempt at simulating extrudate swell of a Maxwell fluid. Die swell of an Oldroyd-B fluid was solved by Crochet and Keunings [6] in 1982. A number of free surface viscoelastic flows were then simulated and studied by Keunings and co-workers [7-10] in the late 1980s. The problem of transient filament stretching was simulated by Kolte et al. [11] by using a Lagrangian approach. Yao and McKinley [12] treated the problem of transient extensional deformation. Cormenzana et al. [13] extended the CONNFESSITT idea [14] to the calculation of free surface flows. More recently Tomé et al. [15] extended GENSMAC, a free surface fluid flow code based on the MAC idea, to viscoelastic flows and solved a number of viscoelastic free surface problems including impacting drops. The methods used in all the works mentioned above are loosely based upon grid-based numerical methods such as FDM and FEM and the techniques mentioned in the previous paragraph for treating moving

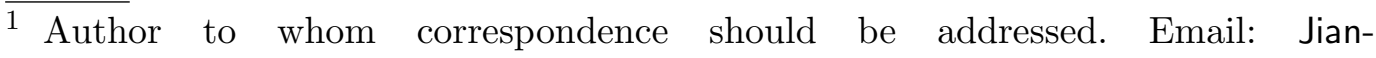
nong.Fang@epfl.ch
} 
free surfaces.

Recently, in the general area of computational mechanics there is a growing interest in developing so-called meshless/meshfree methods or particle methods as alternatives to traditional grid-based methods. Among the various meshfree and particle methods, Smoothed Particle Hydrodynamics (SPH) is the longest established and is quickly approaching a mature stage. SPH is a Lagrangian meshless method in which the problem to be solved is discretized using particles that are free to move rather than by fixed grids or meshes. The governing PDEs are converted into equations of motion for these particles. SPH has several advantages over grid-based methods. Amongst these we highlight the following:

- It handles convection dominated flows and large deformation problems very well due to its Lagrangian and adaptive nature,

- Complex free surfaces, including those with fragmentation, are modeled naturally without the need of any form of explicit surface tracking,

- Complicated physics such as multi-phase, realistic equations of state, compressibility, radiation and solidification can be added easily,

- It is easily able to handle complex geometries in two and three dimensions,

- Programming for complex problems is a comparatively easy task.

Another unique and attractive characteristic of the SPH method is the harmonic combination of the Lagrangian formulation and particle approximation which makes the SPH particles not only function as interpolation points like the particles in other mesh-free methods but also carry material properties.

The SPH method was originally developed for astrophysical applications $[16,17]$. Since its invention, it has been extensively studied, extended and applied in many areas such as the dynamic response of elasto-plastic materials [18-20], free surface flows [21], low-Reynolds number viscous flows [22-24], solid friction [25], incompressible fluids [26,27], heat transfer [28], multi-phase flows [29,30], geophysical flows [31-33] and turbulence modeling [34]. Solid friction has often been studied using embedded atom methods, a method very similar in spirit to SPH [35]. It was not until 2002 that the first application of SPH for the simulation of viscoelastic flows was attempted by Ellero et al. [36], where a corotational Jaumann-Maxwell model was used to describe the viscoelastic behavior of the fluid and a particularly simple time-dependent problem of the viscoelastic relaxation in a $2 \mathrm{D}$ channel was solved by SPH. Recently, Ellero and Tanner [37] assessed the ability of SPH to simulate, stably and accurately, transient viscoelastic problems in comparison with established grid-based Eulerian techniques. In particular, the start-up flow between parallel plates was simulated for an Oldroyd-B and an upper-convected Maxwell (UCM) fluid at low Reynolds number. The results were found to be in good agreement with available analytical solutions. More recently, Ellero et al. [38] 
applied their modified SPH simulation method [36] to the investigation of sheared viscoelastic materials containing rigid non-rotating cylindrical inclusions. To our knowledge, no SPH applications to viscoelastic free surface flows have appeared in the literature to date. For more information on the SPH method, we refer the reader to the new book by Liu and Liu [39] and the most recent review of the method by Monaghan [40].

The motivation of this paper is to extend and test the SPH method for the simulation of viscoelastic free surface flows. In Section 2 the governing equations for the flow of a viscoelastic fluid are outlined. In Section 3 we detail the SPH discretization of the governing equations. Special issues including artificial viscosity, tensile instability and artificial stress, boundary conditions and time marching are discussed in subsections therein. As a typical example of viscoelastic free surface flows, the problem of an impacting drop of an Oldroyd-B fluid is studied in Section 4 and is used to validate the SPH method by comparing its results with the results of a Newtonian fluid and those obtained from a grid-based method. The paper ends with some concluding remarks in Section 5 .

\section{Governing equations}

In a Lagrangian frame, the continuity equation and $\alpha$-th component of the equation of motion for isothermal flow of a viscoelastic fluid may be written as

$$
\begin{gathered}
\frac{D \rho}{D t}=-\rho \frac{\partial v^{\beta}}{\partial x^{\beta}}, \\
\frac{D v^{\alpha}}{D t}=\frac{1}{\rho} \frac{\partial \sigma^{\alpha \beta}}{\partial x^{\beta}}+F^{\alpha},
\end{gathered}
$$

where $\rho$ denotes the fluid density, $v^{\beta}$ the $\beta$-th component of the fluid velocity and $\sigma^{\alpha \beta}$ the $(\alpha, \beta)$-th component of the total (Cauchy) stress tensor. The spatial coordinates $x^{\alpha}$ and time $t$ are the independent variables. In the above two equations, the summations are taken over repeated indices, $D / D t$ is the material (total) derivative defined in a fixed Eulerian frame by

$$
\frac{D}{D t}=\frac{\partial}{\partial t}+v^{\beta} \frac{\partial}{\partial x^{\beta}}
$$

and $F^{\alpha}$ is the $\alpha$-th component of the acceleration due to external forces (for the gravity force considered here $F^{\alpha}=g^{\alpha}$ ).

The total stress tensor in Eq. (2) is made up of two parts, one part consists of an isotropic pressure $p$ and the other part of the extra-stress tensor $\tau$ :

$$
\sigma^{\alpha \beta}=-p \delta^{\alpha \beta}+\tau^{\alpha \beta}
$$


where $\delta^{\alpha \beta}=1$ if $\alpha=\beta$ and $\delta^{\alpha \beta}=0$ if $\alpha \neq \beta$.

To close the system of equations (1)-(2), we require constitutive equations for $p$ and $\boldsymbol{\tau}$. For the Oldroyd-B fluid considered in this paper, the constitutive equation for $\boldsymbol{\tau}$ is given by

$$
\tau^{\alpha \beta}+\lambda_{1} \tau^{\nabla \beta}=\eta\left(d^{\alpha \beta}+\lambda_{2} d^{\nabla \beta}\right),
$$

where the upper convected derivative $\tau^{\stackrel{\nabla}{\alpha \beta}}$ is defined by

$$
\tau^{\nabla \beta}=\frac{D \tau^{\alpha \beta}}{D t}-\frac{\partial v^{\alpha}}{\partial x^{\gamma}} \tau^{\gamma \beta}-\frac{\partial v^{\beta}}{\partial x^{\gamma}} \tau^{\alpha \gamma}
$$

and

$$
d^{\alpha \beta}=\frac{\partial v^{\alpha}}{\partial x^{\beta}}+\frac{\partial v^{\beta}}{\partial x^{\alpha}},
$$

is the rate of strain tensor. In Eq. (5) $\lambda_{1}$ is the time constant of relaxation, $\lambda_{2}$ the time constant of retardation and $\eta$ the total solution viscosity. It is readily seen that by making $\lambda_{2}=0$, we obtain the Upper Convected Maxwell model. The extra-stress tensor $\boldsymbol{\tau}$ may be split in the usual way into

$$
\tau^{\alpha \beta}=\eta\left(\frac{\lambda_{2}}{\lambda_{1}}\right) d^{\alpha \beta}+S^{\alpha \beta}
$$

where $S^{\alpha \beta}$ represents the non-Newtonian (elastic) contribution to the extrastress tensor. By introducing (8) into (5), we then get

$$
S^{\alpha \beta}+\lambda_{1} \stackrel{\nabla}{S^{\alpha \beta}}=\eta\left(1-\frac{\lambda_{2}}{\lambda_{1}}\right) d^{\alpha \beta} .
$$

Many previous works using the SPH method to model incompressible flow have treated the incompressible fluids as being slightly compressible by adopting a specially designed equation of state. In this paper, we follow the same approach by using the following equation of state:

$$
p(\rho)=c^{2}\left(\rho-\rho_{0}\right),
$$

where $c$ is the speed of sound and $\rho_{0}$ is a reference density. It can be shown [21] that the density variation is proportional to the square of the Mach number $M^{2}(M \equiv V / c$, where $\mathrm{V}$ is a typical reference velocity). Therefore, a suitable choice of $c$ can produce the desired density variation. For the simulations performed in this paper, we control the density variation to within $1 \%$ so that the behavior of the artificial compressible fluid is sufficiently close to the real incompressible fluid. 


\section{SPH formulations}

In the SPH method, the fluid is discretised into a finite number of "particles", which possess individual material properties and move according to the governing equations. The relevant physical quantities on each particle are calculated by an interpolation process over neighboring particles. The interpolation process is based on the integral representation of a field function $f(\boldsymbol{x})$,

$$
\langle f(\boldsymbol{x})\rangle=\int_{\Omega} f\left(\boldsymbol{x}^{\prime}\right) W\left(\boldsymbol{x}-\boldsymbol{x}^{\prime}, h\right) d \boldsymbol{x}^{\prime}
$$

where $W$ is the so-called smoothing function or kernel function and $h$ is the smoothing length defining the influence area of $W$. The integral representation converges when $\mathrm{W}$ satisfies the following conditions:

$$
\int_{\Omega} W\left(\boldsymbol{x}-\boldsymbol{x}^{\prime}, h\right) d \boldsymbol{x}^{\prime}=1
$$

and

$$
\lim _{h \rightarrow 0} W\left(\boldsymbol{x}-\boldsymbol{x}^{\prime}, h\right)=\delta\left(\boldsymbol{x}-\boldsymbol{x}^{\prime}\right) .
$$

Moreover, to be valid, it is often required that

$$
W\left(\boldsymbol{x}-\boldsymbol{x}^{\prime}, h\right)>0 \quad \text { over } \quad \Omega
$$

and

$$
W\left(\boldsymbol{x}-\boldsymbol{x}^{\prime}, h\right)=0 \quad \text { when } \quad\left|\boldsymbol{x}-\boldsymbol{x}^{\prime}\right|>k h,
$$

where $k$ is a constant which is a measure of the effective (non-zero) area of the smoothing function centered at a point having position vector $\boldsymbol{x}$. This effective area is called the support domain of the point $\boldsymbol{x}$. Using the compact condition (15), integration over the entire problem domain is localized as the integration over the support domain of a given point. If the smoothing function $W$ is an even function in $\boldsymbol{x}$, by using the Taylor series expansion of $f\left(\boldsymbol{x}^{\prime}\right)$ around $\boldsymbol{x}$, it can be shown that, with the normalization condition (12), the integral representation of $f(\boldsymbol{x})$ is of second order $\left(h^{2}\right)$ accuracy.

The continuous integral representation (11) can be discretized as a summation over all the particles in the support domain as follows:

$$
\langle f(\boldsymbol{x})\rangle \simeq \sum_{j} \frac{m_{j}}{\rho_{j}} f_{j} W\left(\boldsymbol{x}-\boldsymbol{x}_{j}, h\right)
$$

where $m_{j}$ and $\rho_{j}$ are the mass and density of the particle $j$ respectively $\left(m_{j} / \rho_{j}\right.$ gives the volume $V_{j}$ associated with the particle $j$ ) and $j=1,2, \ldots, N$ in which $N$ is the number of particles within the support domain of particle $\boldsymbol{x}$.

The approximation for the spatial derivative $\partial f(\boldsymbol{x}) / \partial \boldsymbol{x}$ can be obtained simply by substituting $f(\boldsymbol{x})$ with $\partial f(\boldsymbol{x}) / \partial \boldsymbol{x}$ in Eq. (11). After integrating by parts 
and using the divergence theorem, this leads to

$$
\left\langle\frac{\partial f(\boldsymbol{x})}{\partial \boldsymbol{x}}\right\rangle=\int_{S} f\left(\boldsymbol{x}^{\prime}\right) W\left(\boldsymbol{x}-\boldsymbol{x}^{\prime}, h\right) \boldsymbol{n} d S-\int_{\Omega} f\left(\boldsymbol{x}^{\prime}\right) \frac{\partial W\left(\boldsymbol{x}-\boldsymbol{x}^{\prime}, h\right)}{\partial \boldsymbol{x}^{\prime}} d \boldsymbol{x}^{\prime}
$$

The first term on the right-hand side of Eq. (17) is the so-called residual boundary integral, which is zero when the support domain is located within the problem domain due to the compact condition (15) for $W$. However, it is normally no longer zero when the support domain is truncated by the boundary of the problem domain. Under such circumstances, boundary effects must be taken into account if the residual boundary integration is treated as zero in Eq. (17) as a convention in SPH. This will be discussed further in section 3.3. By dropping the first term in Eq. (17) and using summation over particles to replace continuous integration in the second term in Eq. (17) as was done in deriving Eq. (16) from Eq. (11), we obtain

$$
\left\langle\frac{\partial f(\boldsymbol{x})}{\partial \boldsymbol{x}}\right\rangle \simeq-\sum_{j} \frac{m_{j}}{\rho_{j}} f_{j} \frac{\partial W\left(\boldsymbol{x}-\boldsymbol{x}_{j}, h\right)}{\partial \boldsymbol{x}_{j}}
$$

The particle approximation for a function and its derivatives at particle $i$ can finally be written in condensed form as

$$
\begin{gathered}
f_{i}=\sum_{j} \frac{m_{j}}{\rho_{j}} f_{j} W_{i j} \\
\left(\frac{\partial f}{\partial \boldsymbol{x}}\right)_{i}=\sum_{j} \frac{m_{j}}{\rho_{j}} f_{j} \frac{\partial W_{i j}}{\partial \boldsymbol{x}_{i}}
\end{gathered}
$$

where $W_{i j}=W\left(\boldsymbol{x}_{i}-\boldsymbol{x}_{j}, h\right)$ and $\partial W_{i j} / \partial \boldsymbol{x}_{i}=\partial W\left(\boldsymbol{x}_{i}-\boldsymbol{x}_{j}, h\right) / \partial \boldsymbol{x}_{i}$. It should be noted that the gradient $\partial W_{i j} / \partial \boldsymbol{x}_{i}$ is calculated with respect to $\boldsymbol{x}_{i}$ not $\boldsymbol{x}_{j}$ as in Eq. (18), so the negative sign in Eq. (18) is removed in Eq. (20). Because $W_{i j}$ is a function of the distance $r=\left|\boldsymbol{x}_{i}-\boldsymbol{x}_{j}\right|$ between the particles $i$ and $j$, we have

$$
\frac{\partial W_{i j}}{\partial \boldsymbol{x}_{i}}=\left(\frac{\boldsymbol{x}_{i}-\boldsymbol{x}_{j}}{r}\right) \frac{\partial W_{i j}}{\partial r} .
$$

The smoothing function is one of the most important ingredients of the SPH method. Its choice is related to the accuracy, efficiency, and stability of the resulting algorithm. In the literature many possible forms for it have been proposed ranging from Gaussian functions to spline functions with the compact condition. In this paper we chose the cubic spline function as the smoothing 
function. Writing $q=r / h$ and choosing $k$ in Eq. (15) equal to 2, it reads

$$
W_{i j}=W(r, h)=w_{0} \times \begin{cases}\frac{2}{3}-q^{2}+\frac{1}{2} q^{3}, & 0 \leq q<1 \\ \frac{1}{6}(2-q)^{3}, & 1 \leq q<2 \\ 0, & q \geq 2,\end{cases}
$$

where the normalization factor $w_{0}=15 /\left(7 \pi h^{2}\right)$ in two-dimensional problems.

The velocity gradients can be calculated as

$$
\kappa_{i}^{\alpha \beta}=\left(\frac{\partial v^{\alpha}}{\partial x^{\beta}}\right)_{i}=\sum_{j} \frac{m_{j}}{\rho_{j}}\left(v_{j}^{\alpha}-v_{i}^{\alpha}\right) \frac{\partial W_{i j}}{\partial x_{i}^{\beta}}
$$

where Eq. (20) is applied directly to evaluate the gradients and the following identity

$$
\left(\frac{\partial 1}{\partial x^{\beta}}\right)_{i}=\sum_{j} \frac{m_{j}}{\rho_{j}} \frac{\partial W_{i j}}{\partial x_{i}^{\beta}}=0
$$

is used. Eq. (23) introduces a velocity difference into the discrete particle approximation in order to ensure that the gradients of a constant velocity field vanish and that the errors arising from the particle inconsistency problem are reduced.

Different transformations or operations on the right-hand side of the continuity equation (1) may lead to different discretized forms of the SPH equation for evolving the density. By applying the following identity to place the density inside the gradient operator

$$
-\rho \frac{\partial v^{\beta}}{\partial x^{\beta}}=-\left(\frac{\partial\left(\rho v^{\beta}\right)}{\partial x^{\beta}}-v^{\beta} \frac{\partial \rho}{\partial x^{\beta}}\right)
$$

and with the gradients evaluated by the approximation (20) and the velocity in the last term on the right-hand side of (25) evaluated at the particle $i$, a popular form of SPH equation for density is obtained as

$$
\frac{D \rho_{i}}{D t}=\sum_{j} m_{j} v_{i j}^{\beta} \frac{\partial W_{i j}}{\partial x_{i}^{\beta}},
$$

where $v_{i j}^{\beta}=v_{i}^{\beta}-v_{j}^{\beta}$. Similarly, a commonly used SPH discretization of the momentum equation is derived as

$$
\frac{D v_{i}^{\alpha}}{D t}=\sum_{j} m_{j}\left(\frac{\sigma_{i}^{\alpha \beta}}{\rho_{i}^{2}}+\frac{\sigma_{j}^{\alpha \beta}}{\rho_{j}^{2}}\right) \frac{\partial W_{i j}}{\partial x_{i}^{\beta}}+g^{\alpha},
$$


by considering the following identity

$$
\frac{1}{\rho} \frac{\partial \sigma^{\alpha \beta}}{\partial x^{\beta}}=\frac{\partial\left(\sigma^{\alpha \beta} / \rho\right)}{\partial x^{\beta}}+\frac{\sigma^{\alpha \beta}}{\rho^{2}} \frac{\partial \rho}{\partial x^{\beta}},
$$

and applying the SPH particle approximation to the gradients.

The SPH discretization of the constitutive equation (9) for the elastic stress tensor is straightforward and reads

$$
\frac{D S_{i}^{\alpha \beta}}{D t}=\kappa_{i}^{\alpha \gamma} S_{i}^{\gamma \beta}+\kappa_{i}^{\beta \gamma} S_{i}^{\alpha \gamma}-\frac{1}{\lambda_{1}} S_{i}^{\alpha \beta}+\frac{\eta}{\lambda_{1}}\left(1-\frac{\lambda_{2}}{\lambda_{1}}\right) d_{i}^{\alpha \beta},
$$

where $d_{i}^{\alpha \beta}=\left(\kappa_{i}^{\alpha \beta}+\kappa_{i}^{\beta \alpha}\right)$ and $\kappa_{i}^{\alpha \beta}$ is given by Eq. (23).

Finally, the particle positions are moved consistently according to

$$
\frac{d x_{i}^{\alpha}}{d t}=v_{i}^{\alpha} .
$$

\subsection{Artificial viscosity}

Most implementations of SPH employ an artificial viscosity to allow SPH to be capable of modeling shock waves or simply to stabilize a numerical scheme. Many forms of artificial viscosity have been proposed. The most commonly used one [41] is obtained by writing the term in the parentheses of Eq. (27) as

$$
\frac{\sigma_{i}^{\alpha \beta}}{\rho_{i}^{2}}+\frac{\sigma_{j}^{\alpha \beta}}{\rho_{j}^{2}}+\Pi_{i j} \delta^{\alpha \beta} .
$$

The detailed formulation of the artificial viscosity is as follows:

$$
\Pi_{i j}=\frac{-\alpha_{\Pi} \bar{c}_{i j} \phi_{i j}+\beta_{\Pi} \phi_{i j}^{2}}{\bar{\rho}_{i j}} \quad \text { for } \quad \boldsymbol{v}_{i j} \cdot \boldsymbol{x}_{i j}<0,
$$

and

$$
\Pi_{i j}=0 \quad \text { for } \quad \boldsymbol{v}_{i j} \cdot \boldsymbol{x}_{i j} \geq 0
$$

where

$$
\begin{gathered}
\phi_{i j}=\frac{h \boldsymbol{v}_{i j} \cdot \boldsymbol{x}_{i j}}{\left|\boldsymbol{x}_{i j}\right|^{2}+0.01 h^{2}}, \\
\bar{c}_{i j}=\left(c_{i}+c_{j}\right) / 2, \\
\bar{\rho}_{i j}=\left(\rho_{i}+\rho_{j}\right) / 2, \\
\boldsymbol{v}_{i j}=\boldsymbol{v}_{i}-\boldsymbol{v}_{j}, \\
\boldsymbol{x}_{i j}=\boldsymbol{x}_{i}-\boldsymbol{x}_{j} .
\end{gathered}
$$


In the above equations, $\alpha_{\Pi}$ and $\beta_{\Pi}$ are constants that are all typically set approximately equal to 1 . The $0.01 h^{2}$ term is included to prevent numerical divergence when two particles are approaching each other. SPH simulations with variable smoothing length $h$ can be also performed [39]. In the artificial viscosity, the first term associated with $\alpha_{\Pi}$ involves shear and bulk viscosity, while the second term associated with $\beta_{\Pi}$ is similar to the von NeumannRichtmeyer viscosity for resolving shocks and is very important in preventing unrealistic particle penetration, especially for particles that are approaching each other at high speed. For flows with physical viscosity implemented by the approach of nested summation [42] (adopted in this paper) and by other approaches [24], retaining the first term in $\Pi_{i j}$ results in larger shear viscosity. Therefore, it has been proposed that this term be removed by setting $\alpha_{\Pi}=0$ and that the second term be retained to help prevent unphysical particle penetration. However, unlike the case of low-Reynolds number viscous flows, for the viscoelastic free surface flows studied in this paper it is found necessary to keep the first term which helps to keep the particles in order and improve the numerical stability (see section 4.1). For the results reported in this paper, the conventional choice of $\alpha_{\Pi}=1$ and $\beta_{\Pi}=2$ is used as default, except special indication.

\subsection{Tensile instability and artificial stress}

When the SPH method is applied to solids, a numerical problem called tensile instability [43] arises. That is, when the material is in a state of tensile stress (stretched), the motion of the particles becomes unstable. It can result in the SPH particles forming clumps and therefore giving rise to unrealistic fracture behavior of the material. In the worst case, it can lead to complete blowup in the computation. There have been a number of attempts to remove the tensile instability in elastic and elasto-plastic dynamics of solid materials. The most successful has been the artificial stress method $[44,45]$. In Section 4, we will show that the tensile instability problem also occurs in the free surface flow problem studied in this paper for a viscoelastic fluid. Here we follow the artificial stress approach to deal with the problem and test to see if it also works for viscoelastic fluids. The key idea of the artificial stress method is to introduce a small short-range repulsive force between a pair of neighboring particles to prevent them from getting too close when the two particles are in a state of tensile stress and thereby subject to attraction due to the original $\mathrm{SPH}$ formulation of the force between them (see Eq. (27)). To do so, we replace the expression (30) with

$$
\frac{\sigma_{i}^{\alpha \beta}}{\rho_{i}^{2}}+\frac{\sigma_{j}^{\alpha \beta}}{\rho_{j}^{2}}+\Pi_{i j} \delta^{\alpha \beta}+f_{i j}^{n}\left(R_{i}^{\alpha \beta}+R_{j}^{\alpha \beta}\right),
$$


where $n$ is an exponential factor and $f_{i j}$ is defined by the ratio

$$
f_{i j}=\frac{W_{i j}}{W(\Delta d, h)}
$$

with $\Delta d$ being the initial distance between two neighboring particles along the coordinate axes. In this paper, $h$ is always assumed to be constant and so $W(\Delta d, h)$ is also constant. For the cubic spline function (22) the ratio $W(0) / W(\Delta d)$ has the value 1.8 if $h=1.5 \Delta d$ (the typical $h$ used in this paper). The exponential factor $n$ is chosen to be 4 so that the repulsive force increases by a factor of about 10 as $r$ decreases from $\Delta d$ to zero and $f_{i j}^{n}$ decreases rapidly in the domain $h \leq r \leq 2 h$. This ensures that only the nearest neighbors are significantly affected by the artificial stress. For a $2 \mathrm{D}$ problem with planar Cartesian coordinates $(x, y)$, the components of the artificial stress tensor $R^{\alpha \beta}$ are constructed by means of the following transformation relations

$$
\begin{aligned}
& R^{x x}=R^{\prime x x} \cos ^{2} \theta+R^{\prime y y} \sin ^{2} \theta, \\
& R^{y y}=R^{\prime x x} \sin ^{2} \theta+R^{\prime y y} \cos ^{2} \theta, \\
& R^{x y}=\sin \theta \cos \theta\left(R^{\prime x x}-R^{\prime y y}\right),
\end{aligned}
$$

where $R^{\prime x x}$ and $R^{\prime y y}$ are the diagonal components of the artificial stress tensor along the principal axes $x^{\prime}$ and $y^{\prime}$, respectively. The coordinate frame $\left(x^{\prime}, y^{\prime}\right)$ is rotated with respect to $(x, y)$ by an angle $\theta$ given by

$$
\theta=\frac{1}{2} \tan ^{-1}\left(\frac{2 \sigma^{x y}}{\sigma^{x x}-\sigma^{y y}}\right)
$$

such that the stress tensor in the rotated frame is diagonal. Note that $\sigma^{x x}$, $\sigma^{y y}$, and $\sigma^{x y}$ are the components of the stress tensor in the original frame. The expression for $R^{\prime x x}$ was given by Gray et al. [45] as the following

$$
R^{\prime x x}=\left\{\begin{aligned}
-\epsilon \frac{\sigma^{\prime x x}}{\rho^{2}} & \text { if } \sigma^{\prime x x}>0 \\
0 & \text { otherwise }
\end{aligned}\right.
$$

In Eq. (42), $\epsilon$ is a small parameter with value between 0 and 1 . An identical form applies to $R^{\prime y y}$. The diagonal components $\sigma^{\prime x x}$ and $\sigma^{\prime y y}$ of the stress tensor in the rotated frame can be calculated in terms of those in the original frame through the relations

$$
\begin{aligned}
& \sigma^{\prime x x}=\cos ^{2} \theta \sigma^{x x}+2 \cos \theta \sin \theta \sigma^{x y}+\sin ^{2} \theta \sigma^{y y}, \\
& \sigma^{\prime y y}=\sin ^{2} \theta \sigma^{x x}-2 \cos \theta \sin \theta \sigma^{x y}+\cos ^{2} \theta \sigma^{y y} .
\end{aligned}
$$




\subsection{Boundary conditions}

In order to solve the system of equations (1), (2) and (9) one needs to impose appropriate boundary conditions. We here consider two types of boundaries: a free surface and a rigid wall. On free surfaces, we specify the stress-free condition

$$
\boldsymbol{\sigma} \cdot \boldsymbol{n}=0,
$$

by neglecting surface tension and taking the pressure of the atmosphere into which the fluid moves to be zero. In the above equation $\boldsymbol{n}$ denotes a unit normal vector to the surface. The stress-free condition (45) is satisfied naturally by the SPH summation (18) in evaluating the stress gradient in the momentum equation (2) because it is represented in the residual boundary integration (see Eq. (17)) which is originally neglected in the SPH approximations for derivatives and happens to be zero in this case. However, when dealing with boundary conditions on rigid walls, the SPH method may encounter the problem of particle deficiency near or on the boundary, which results from the integral that is truncated by the boundary. For particles near or on the boundary, only particles inside the boundary contribute to the SPH summation for the gradient calculation and there is no contribution from outside. The one-sided contribution causes inaccurate solutions. A natural attempt at solving the problem is to revert to the original integral estimate and utilise the boundary conditions through the residual boundary integration, which are only non-zero for particles whose supporting domains include a boundary. Campbell [46] first tried this idea, but no numerical results were reported. Our experience in testing the idea is that although it has worked in some cases (including lid-driven cavity flow and dam collapse), a numerical instability was found in the simulation of Poiseuille flow between two parallel plates. The reason for this needs further investigation. So, common practice in solving the problem of particle deficiency is still to use the various techniques based on virtual (or, ghost) particles [22,24,47].

In this paper, two types of virtual particles are used to implement the boundary conditions on a straight solid wall. The virtual particles of the first type are located right on the solid boundary, hence we name them as "boundary particles". In contrast to the work by Monaghan [21], boundary particles in our work do not exert an artificial repulsive force on approaching real particles to prevent them from penetrating solid walls. Instead, as in the work of Morris et al. [24], boundary particles contribute to the usual SPH expressions for velocity, pressure and stress gradients. However, unlike Morris et al. [24], densities of boundary particles are not evolved, which was found important for predicting more realistically the shape of the free surface near the solid boundary for the impacting problem of viscoelastic drop studied here. Note that boundary particles have the same velocity as the wall (normally zero) but that their positions remain fixed in time even when the wall is moving. 
The pressure and components of the elastic stress on the boundary particles are calculated according to a normalized SPH interpolation for a function $f$, which reads

$$
f_{i}=\frac{\sum_{j} \frac{m_{j}}{\rho_{j}} f_{j} W_{i j}}{\sum_{j} \frac{m_{j}}{\rho_{j}} W_{i j}},
$$

where $i$ represents the index of a specific point corresponding to a boundary particle and $j$ runs over the indices of its neighboring real (fluid) particles only (not boundary particles). The normal component of the elastic stress in the direction perpendicular to the wall surface is set to zero. The viscous stress tensor on the boundary particles can be calculated either in the same way as for that on the real particles, by evaluating the velocity gradients using Eq. (23) or using the normalized SPH interpolation (46). For the results presented in this paper, the latter is adopted.

The virtual particles of the second type are placed just outside the solid boundary and fill a domain with at least a range of depth comparable with the support length $2 h$ of the kernel function. These virtual particles are called "image particles" and have fixed densities and positions. The velocity and the elastic stress tensor on the image particles $\left(\boldsymbol{v}_{I}\right.$ and $\left.S_{I}^{\alpha \beta}\right)$ are calculated in the following way: for each image particle $(I)$, a corresponding point $(P)$ just inside the solid boundary with a normal distance to the boundary comparable to the initial smallest separation between real particles is located such that the line connecting $I$ to $P$ is perpendicular to the solid boundary. Then $\boldsymbol{v}_{I}$ and $S_{I}^{\alpha \beta}$ on the image particle are obtained from those on $P\left(\boldsymbol{v}_{P}\right.$ and $\left.S_{P}^{\alpha \beta}\right)$ through the linear extrapolations

$$
\begin{gathered}
\boldsymbol{v}_{I}=\boldsymbol{v}_{P}+\left(1+\frac{d_{I}}{d_{P}}\right)\left(\boldsymbol{v}_{W}-\boldsymbol{v}_{P}\right), \\
S_{I}^{\alpha \beta}=S_{P}^{\alpha \beta}+\left(1+\frac{d_{I}}{d_{P}}\right)\left(S_{W}^{\alpha \beta}-S_{P}^{\alpha \beta}\right),
\end{gathered}
$$

where $\boldsymbol{v}_{W}$ is the wall velocity and $S_{W}^{\alpha \beta}$ is the elastic stress tensor, both evaluated at the point where the line connecting $I$ and $P$ meets the wall. The values of the components of $S_{W}^{\alpha \beta}$ are calculated from those on the two neighboring boundary particles by a linear interpolation. To specify the values for $\boldsymbol{v}_{P}$ and $S_{P}^{\alpha \beta}$, again the normalized SPH interpolation (46) is applied. An identical procedure follows for the calculation of the pressure and viscous stress tensor on the image particles.

The use of boundary and image particles as described above permits us to enforce the no-slip condition on the solid boundary and solves the particle deficiency problem in evaluating velocity and stress gradients on the real particles near the wall. Moreover, since both boundary particles and image particles contribute to the evolution of the density of the real particles, pressures on both real and virtual particles increase when real particles move towards 
the wall. Practically, it is found that this pressure-driven repulsive force alone is strong enough to prevent real particles from penetrating the wall without resorting to an additional artificial repulsive force.

\subsection{Time integration scheme}

To solve the system of ordinary differential equations (26)-(29) along the particle paths, a simple predictor-corrector scheme [21] is adopted. If $\boldsymbol{X}$ represents the vector of variables $\left(\boldsymbol{x}_{i}, \rho_{i}, \boldsymbol{v}_{i}, S_{i}^{\alpha \beta}\right)$ and $\boldsymbol{F}$ the vector of right-hand sides of Eqs. (26)-(29), the predictor step predicts the midpoint value of $\boldsymbol{X}$ so that, with time step $\Delta t$, the midpoint $\boldsymbol{X}^{n+1 / 2}$ is

$$
\boldsymbol{X}^{n+1 / 2}=\boldsymbol{X}^{n}+\boldsymbol{F}^{n} \Delta t / 2
$$

Then in the corrector step, the value of $\boldsymbol{X}$ at the end of one time step is given by

$$
\boldsymbol{X}^{n+1}=\boldsymbol{X}^{n}+\boldsymbol{F}^{n+1 / 2} \Delta t
$$

For numerical stability, several time step constraints must be satisfied, including a Courant-Friedrichs-Lewy (CFL) condition,

$$
\Delta t \leq \frac{h}{c}
$$

and additional constraints due to the hydrodynamical force acting on the particle $F_{a}$,

$$
\Delta t \leq \min _{\forall a}\left(\frac{h}{F_{a}}\right)^{1 / 2}
$$

and viscous diffusion,

$$
\Delta t \leq 0.5 \frac{h^{2}}{\nu}
$$

where $\nu=\eta / \rho_{0}$ is the kinematic viscosity. Eq. (51) states that the maximum speed of numerical propagation must exceed the speed of sound $c$. Eq. (52) is based upon the requirement that the time step is always smaller than the inverse Einstein frequency of the system. Eq. (53) comes from the usual condition for an explicit finite difference method for simulating diffusion. At sufficiently high resolution (sufficiently small $h$ ) or large viscosity as in the problem studied here, Eq. (53) is typically the dominant constraint for the time step. 


\section{Numerical results}

In this section, results from two-dimensional flow simulations of a drop of a viscoelastic fluid impacting a rigid wall

$$
\{(x, y):-\infty<x<\infty, y=0\}
$$

are presented to test the ability of the SPH method presented in this paper to simulate transient viscoelastic free surface flows. The $y$ ordinate in the Cartesian coordinate system that we adopt increases as one moves into the flow domain away from the wall. To highlight the observed viscoelastic effects in the drop, the results of the simulation are compared with those obtained for a Newtonian drop. We consider a drop (a disk in 2D) of a viscoelastic fluid modeled by the Oldroyd-B equations whose initial diameter is $D=2 \mathrm{~cm}$. At $t=0$, the fluid is in a stress-free state, the initial velocity of the drop is $V=-1 \mathrm{~ms}^{-1}$ and the center of the drop has coordinates $(0,4 \mathrm{~cm})$. Gravity acts in the direction of $y$ decreasing and the acceleration due to the gravitational force is taken as $g=-9.81 \mathrm{~ms}^{-2}$. For all results presented in this paper the speed of sound $c$ was set equal to $12.5 \mathrm{~ms}^{-1}$. Boundary conditions on the wall were those described in Section 3.3. The particles comprising the drop were initially set on our Cartesian grid and those particles within the circle defining the circumference were retained. As a result the drop has a slightly roughened appearance. The number of real particles used for the simulation results presented here is 7845 giving a initial nearest neighbor separation $\Delta d=0.02 \mathrm{~cm}$. Additionally, 501 boundary particles are placed on the wall at $y=0 \mathrm{~cm}$ from $x=-5 \mathrm{~cm}$ to $x=5 \mathrm{~cm}$ and three consecutive rows of 501 image particles each are placed on the side just outside the wall within the same range of $x$ as the boundary particles. The placement of the image and boundary particles is shown in Fig. 1. The total number of virtual particles is then 2004 with the particle spacings in the $x$ and $y$ directions $\Delta x=\Delta y=\Delta d$. The "linkedlist" algorithm [48] for searching the nearest neighbor particles was adopted. A time step of $1 \times 10^{-5}$ s was used to ensure numerical stability. Simulations using 31417 particles (corresponding to $\Delta d=0.01 \mathrm{~cm}$ ) were also performed with smaller time steps ranging from $1 \times 10^{-6} \mathrm{~s}$ to $5 \times 10^{-6} \mathrm{~s}$. The obtained results were qualitatively the same as those using 7845 particles and quantitative differences between the two choices were small.

\subsection{Newtonian and Oldroyd-B fluid drops}

For the results presented in Figs. 2-6, the fluid material parameters were chosen to be $\nu=0.004 \mathrm{~m}^{2} \mathrm{~s}^{-1}, \lambda_{1}=0.02 \mathrm{~s}$ and $\lambda_{2}=0.002 \mathrm{~s}$. The adoption of these 


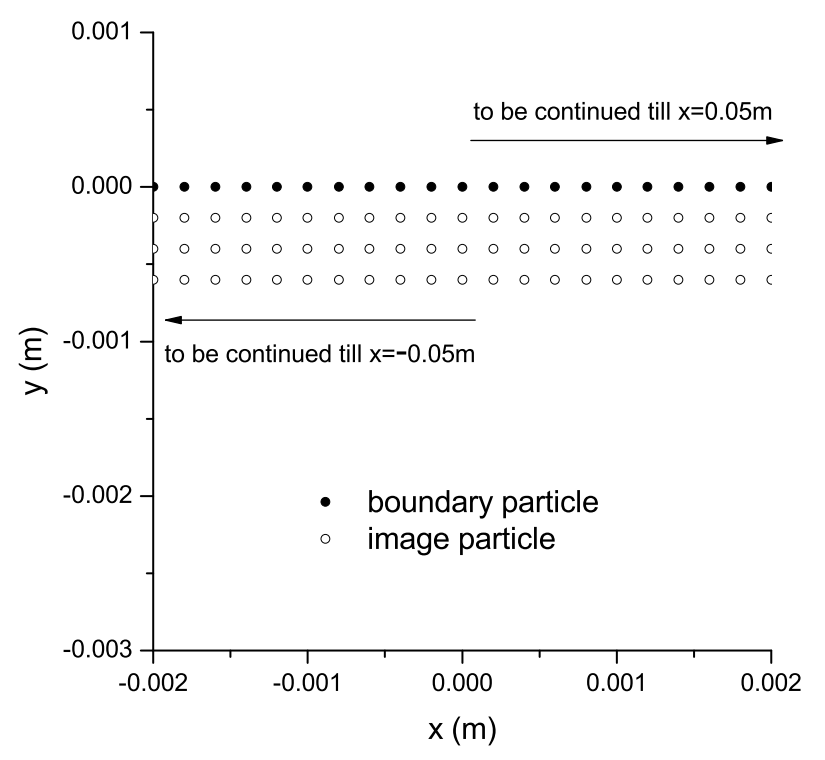

Fig. 1. The placement of the image and boundary particles.

parameter values leads to a Reynolds number

$$
R_{e}=\frac{D V}{\nu}=5
$$

and a Weissenberg number

$$
W_{e}=\frac{\lambda_{1} V}{D}=1
$$

Fig. 2 shows the particle positions of the drop at short times just after impacting the wall for the Newtonian and viscoelastic cases with different values of $\epsilon$. It can be seen that without the artificial stress term $(\epsilon=0)$ the drop fractures unrealistically in the viscoelastic case and the simulation will eventually diverge. In the Newtonian case without the first term of artificial viscosity $\left(\alpha_{\Pi}=0\right)$, fracture is also observable but not as severe as in the viscoelastic case and the simulation can continue. Not shown are the results for the Newtonian case with the first term of artificial viscosity $\left(\alpha_{\Pi}=1\right)$ where fracture is almost not recognizable and an artificial stress is convenient but not necessary. Fracture is demolished totally by increasing the value of $\epsilon$ up to 0.5 for the viscoelastic case and 0.2 for the Newtonian case. It may be concluded that tensile instability is more dominant in viscoelastic fluid than its viscous counterpart.

We also did simulation without the first term of artificial viscosity for the viscoelastic case in which artificial stress is needed anyway to avoid fracture and blowup of simulation. Results are still obtainable and reasonable with the resolution using 7845 particles, but the simulation diverged with the refined resolution using 31417 particle and a smaller time step $5 \times 10^{-6}$ s. By adding back the first term of artificial viscosity, the simulation went well with the 

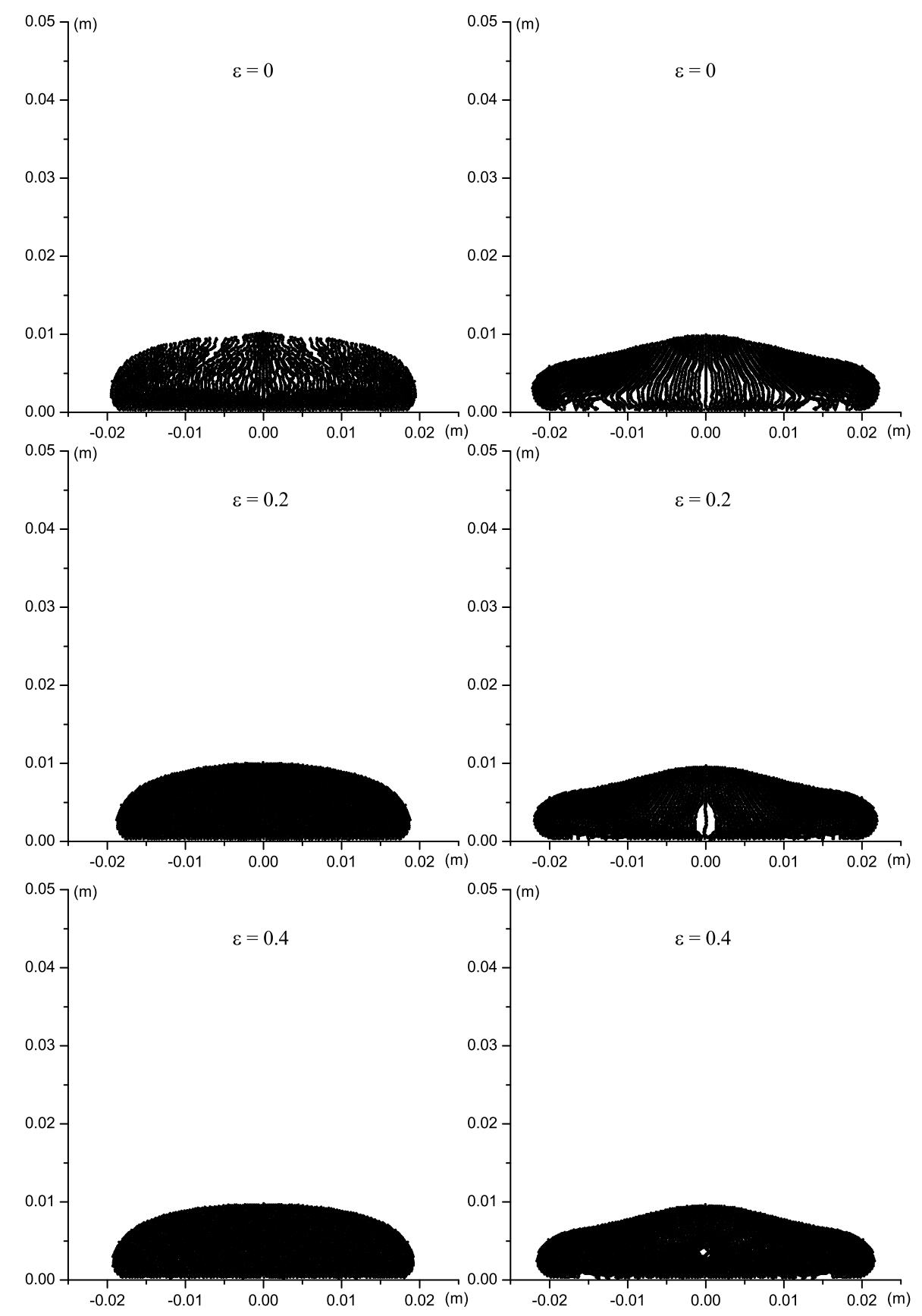

Fig. 2. SPH simulation of a falling drop of a Newtonian (left column) and an Oldroyd-B (right column) fluid towards a rigid wall using different values of $\epsilon$ as indicated. Shown are particle positions at the dimensionless time $t=3.625$ for the Newtonian fluid with $\alpha_{\Pi}=0$ and $\beta_{\Pi}=2$ and $t=2$ for the viscoelastic fluid with $\alpha_{\Pi}=1$ and $\beta_{\Pi}=2$.

same refined resolution in space and time, and the differences between the results of the two resolutions are quite small. Therefore, it may be concluded that, for the viscoelastic case, the first term of artificial viscosity must be retained for stability reasons also in the case of using artificial stress, and, for 
the Newtonian case, it is optional.

In Figures 3-6 we show the results at different times with $\epsilon=0.5$ for the viscoelastic case and $\epsilon=0.2$ for the Newtonian case. As may be seen from these figures, the Newtonian drop hits the wall and spreads out evenly with its convex shape kept during the whole simulation process. For the viscoelastic drop, the flow process may be divided into three phases. The first phase, between the time the drop hits the wall and the dimensionless time $t=2.5$, is associated with a negative vertical velocity. In this phase the viscoelastic flow demonstrates a greater tendency to spread horizontally than its Newtonian counterpart. From $t=2.5$ to $t=3.75$ is the second phase which may be linked with a positive vertical velocity. During this phase, the drop contracts because of the elasticity of the fluid. It is found that an indentation is first formed and then a dome is formed at the center of the domain. Finally, in the third phase where $t>3.75$, elastic effects have been damped out and the drop slowly spreads out in a manner similar to that of the Newtonian drop.

\subsubsection{Comparison with the results of Tomé et al. [15]}

The qualitative behavior described above has also been predicted using gridbased methods such as the finite difference method (FDM) of Tomé et al. [15]. To evaluate the SPH method quantitatively, the results from the present work and those of Tomé et al. [15] for the evolution of the width of the drop are compared in Figs. 7 and 8 for the Newtonian and Oldroyd-B fluids, respectively. It can be seen from Fig. 7 that the first term of artificial viscosity produces an effective dissipation which slows down the horizontal expanding speed of the Newtonian drop. However the differences between the results of $\alpha_{\Pi}=0$ and $\alpha_{\Pi}=1$ shown in Fig.7. are small. The results of both Newtonian and viscoelastic cases using the SPH method are qualitatively in good agreement with the results from the FDM and differ quantitatively from those obtained with the FDM by a maximum of $10 \%$. The discrepancy could be mainly due to the particle inconsistency problem (low accuracy of the SPH method which does not even have $C^{0}$ and $C^{1}$ consistency in the particle approximation) and artificial compressibility. As a check on the latter, the volume of the drop was calculated. It was found that, for both Newtonian and viscoelastic cases, the volume of the drop slightly decreased over the course of the simulations but that the percentage error in volume varied no more than $4 \%$.

\subsubsection{Higher viscosity drop}

It may be anticipated that with an appropriate choice of material parameters the drop will bounce during the retreating phase. In Fig. 9 we show the results with $\nu=0.04 \mathrm{~m}^{2} \mathrm{~s}^{-1}$ and the other parameters kept the same as those for the 

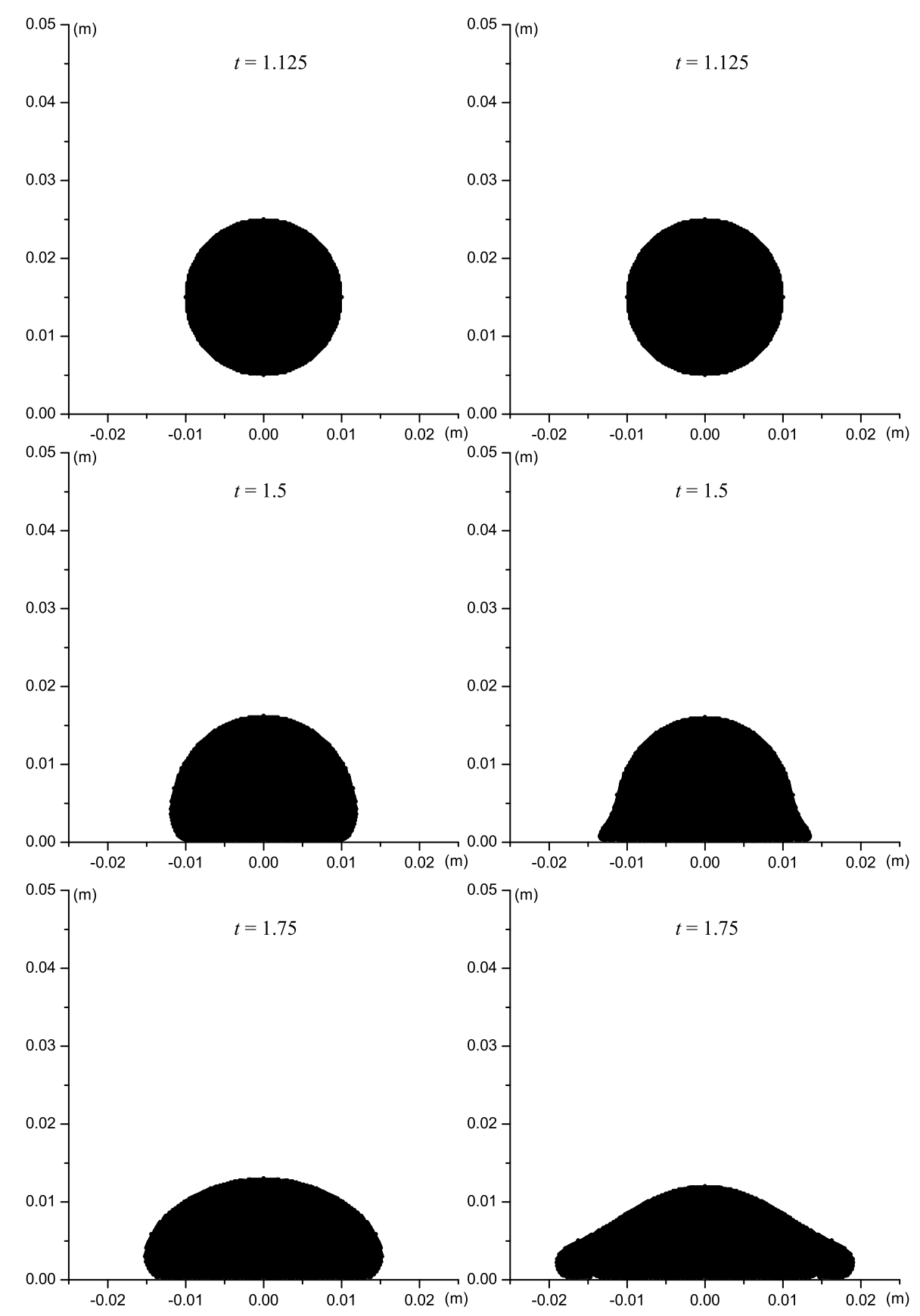

Fig. 3. SPH simulation of a falling drop of a Newtonian ( $\epsilon=0.2$, left column) and an Oldroyd-B ( $\epsilon=0.5$, right column) fluid towards a rigid wall. Fluid flow visualization at different dimensionless times $t$ as indicated.

viscoelastic case in Figs. 3-6. The method proposed is able to capture the bounce phenomena nicely. 

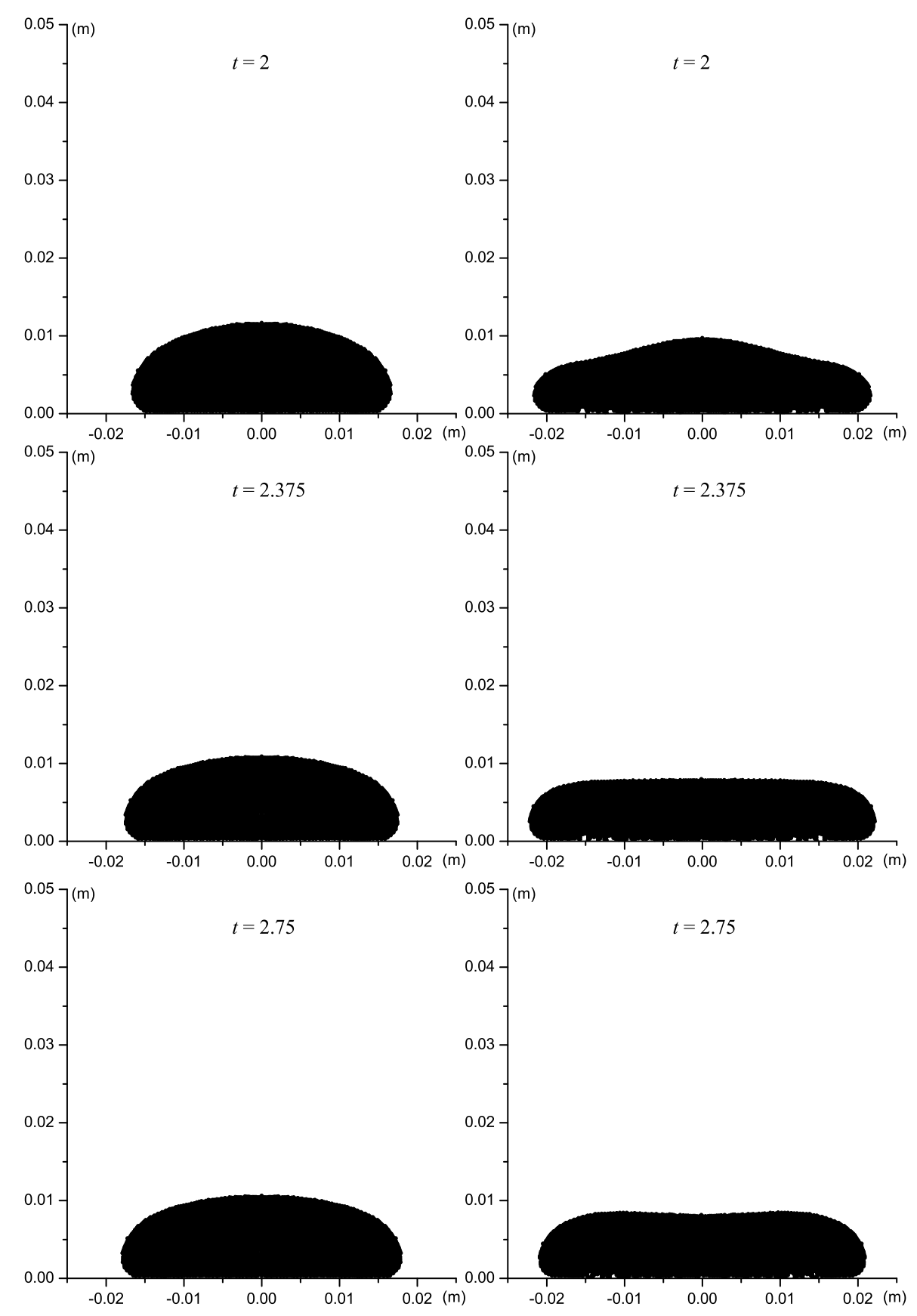

Fig. 4. SPH simulation of a falling drop of a Newtonian ( $\epsilon=0.2$, left column) and Oldroyd-B ( $\epsilon=0.5$, right column) fluid towards a rigid wall. Fluid flow visualization at different dimensionless times $t$ as indicated.

\subsubsection{UCM fluid drop}

The simulations for a drop of a UCM fluid $\left(\lambda_{2}=0\right.$ but all other parameters kept the same as those for the viscoelastic case in Figs. 3-6) were also performed and the results are presented in Fig. 10. The flow process can also be divided into three phases similar to those of the viscoelastic case shown in 

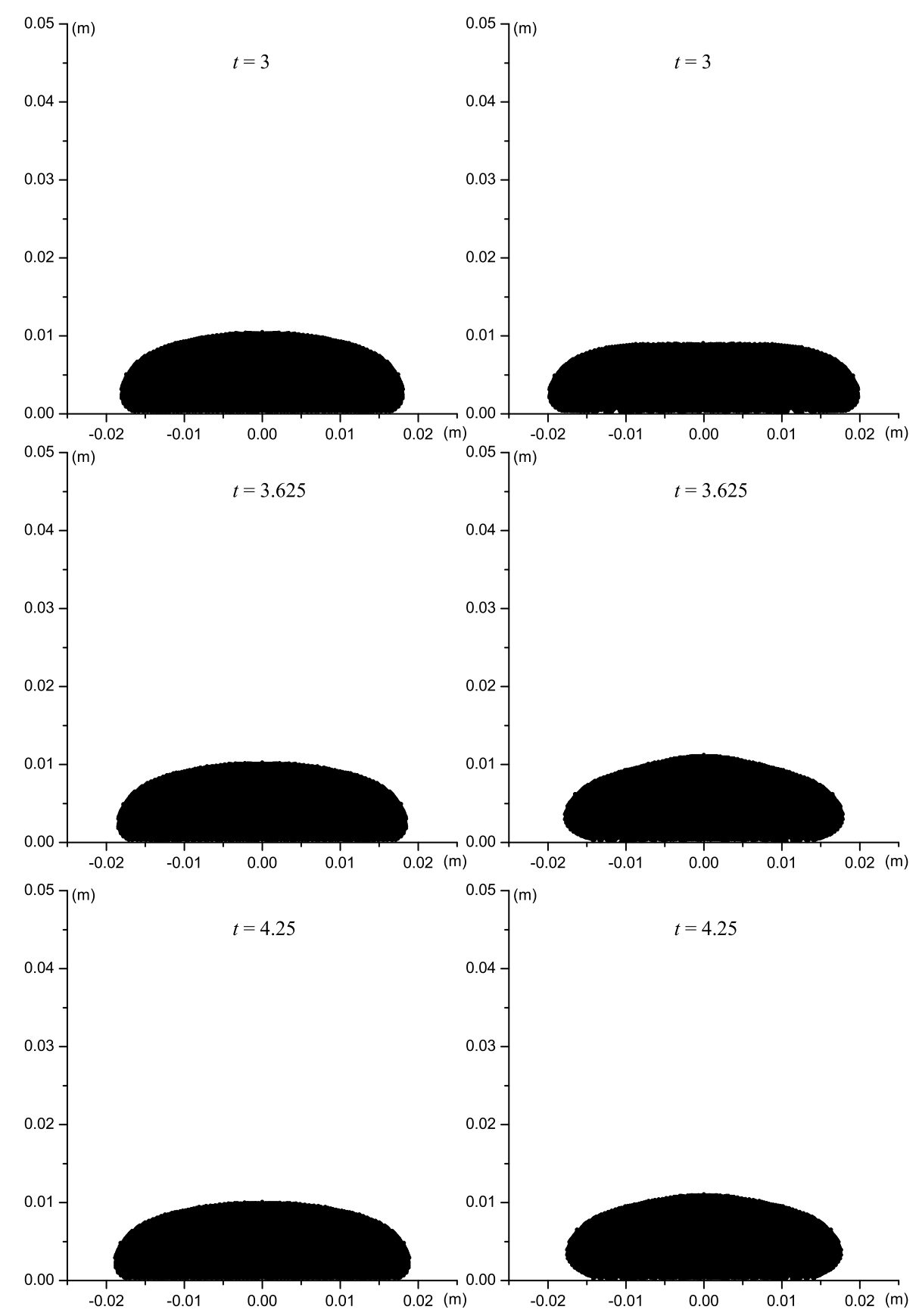

Fig. 5. SPH simulation of a falling drop of a Newtonian ( $\epsilon=0.2$, left column) and Oldroyd-B ( $\epsilon=0.5$, right column) fluid towards a rigid wall. Fluid flow visualization at different dimensionless times $t$ as indicated.

Figs. 3-6. Some qualitative changes in the shape of the drop, especially during the second phase, may be observed. However runs with a refined resolution using 31417 particles (corresponding to $\Delta d=0.01 \mathrm{~cm}$ ) diverged with two smaller time steps $\left(5 \times 10^{-6} \mathrm{~s}\right.$ and $\left.1 \times 10^{-6} \mathrm{~s}\right)$. 

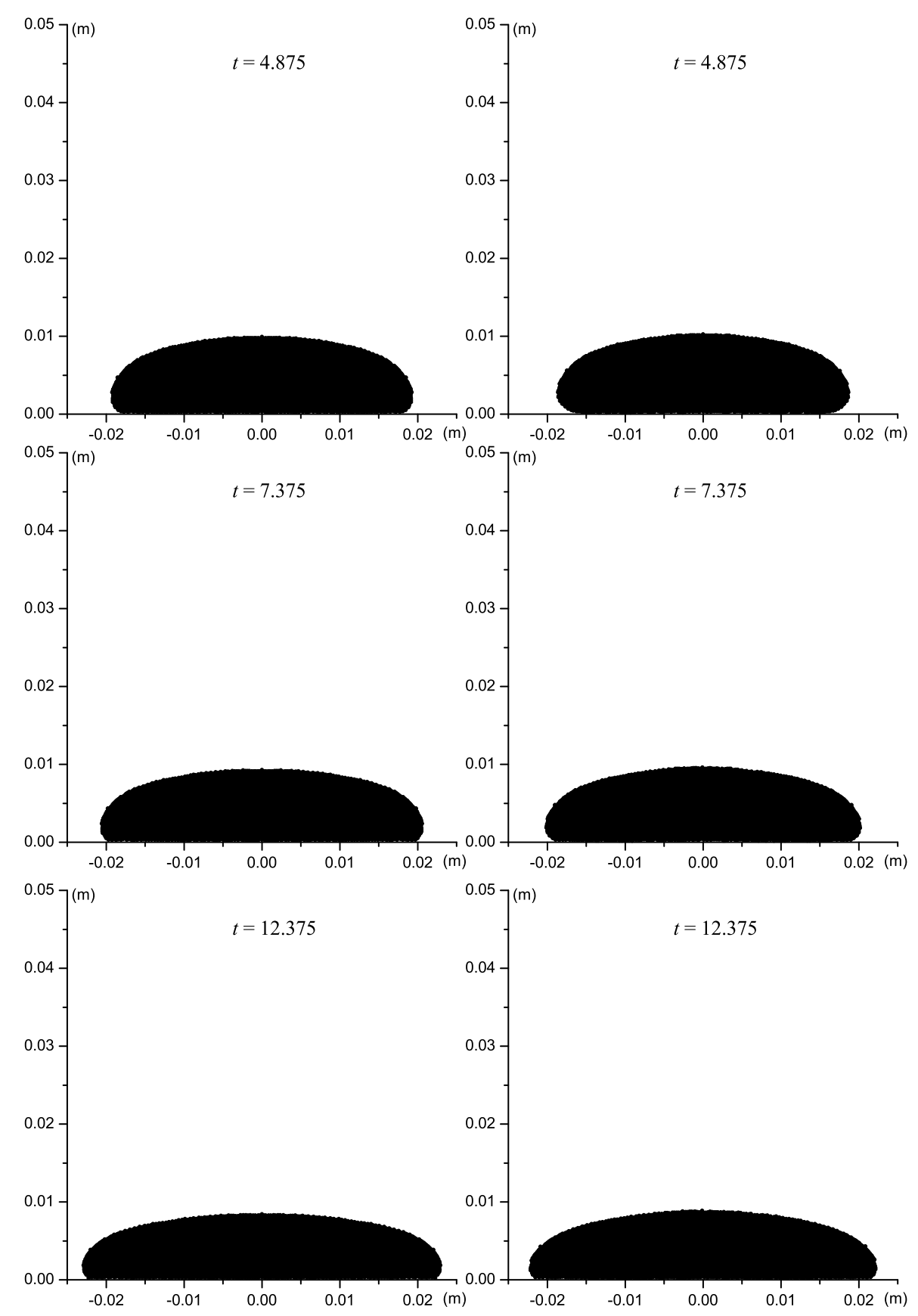

Fig. 6. SPH simulation of a falling drop of a Newtonian ( $\epsilon=0.2$, left column) and an Oldroyd-B $(\epsilon=0.5$, right column) fluid towards a rigid wall. Fluid flow visualization at different dimensionless times $t$ as indicated.

\subsubsection{High Weissenberg number simulations}

Finally, it is often the case that, with increasing relaxation time $\lambda_{1}$ (and hence Weissenberg number $W_{e}$ ), numerical simulations of a viscoelastic fluid can become unstable and may eventually encounter a limit on $W_{e}$, beyond which no mesh-converged solution is possible. To check on this point for the SPH method 


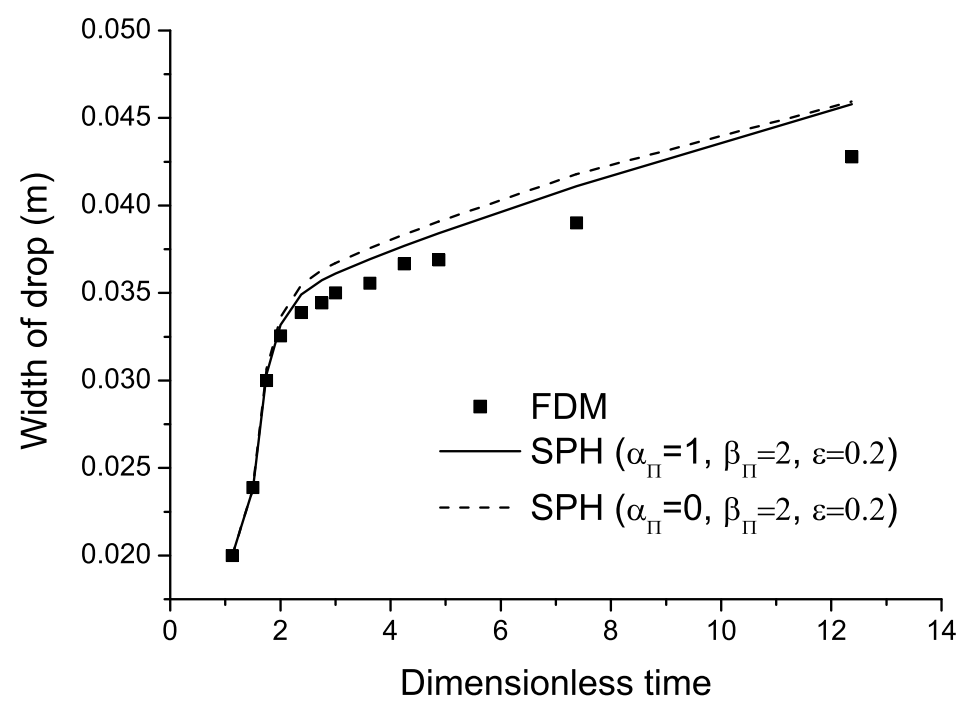

Fig. 7. Comparison of the results obtained using FDM [15] and the SPH method for the free surface flow of a Newtonian drop impacting a rigid wall: width of the drop varying with time.

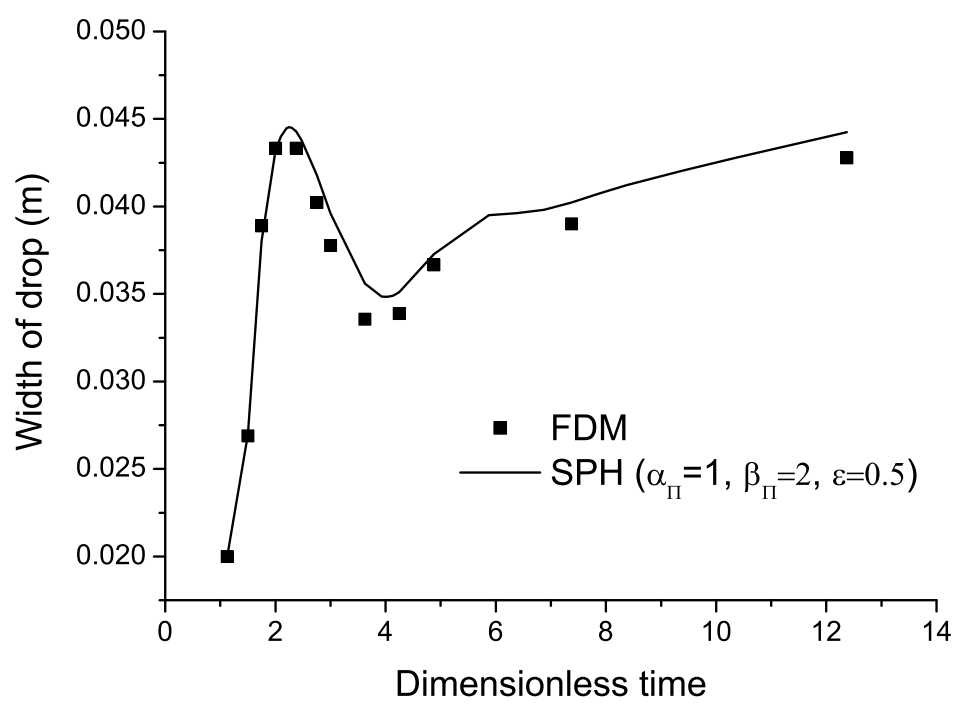

Fig. 8. Comparison of the results obtained using FDM [15] and the SPH method for the free surface flow of an Oldroyd-B drop impacting a rigid wall: width of the drop against time.

studied here, we doubled the relaxation and retardation times to $\lambda_{1}=0.04 \mathrm{~s}$ and $\lambda_{2}=0.004 \mathrm{~s}$, respectively. No numerical problems were encountered in the simulations. Indeed, the results were similar to those shown in Figs. 36 , the only noticeable difference being that the drop spread out to a greater extent in the $x$ direction during the first phase, retreated back at a later time and then extended again. With the ratio $\lambda_{2} / \lambda_{1}$ fixed to 0.1 , simulations were pursued up to Weissenberg numbers of 10 without any numerical difficulties being encountered. However, like the case in subsection 4.1.3, runs for $W_{e}=2$ and $W_{e}=10$ with a refined resolution using 31417 particles (corresponding 

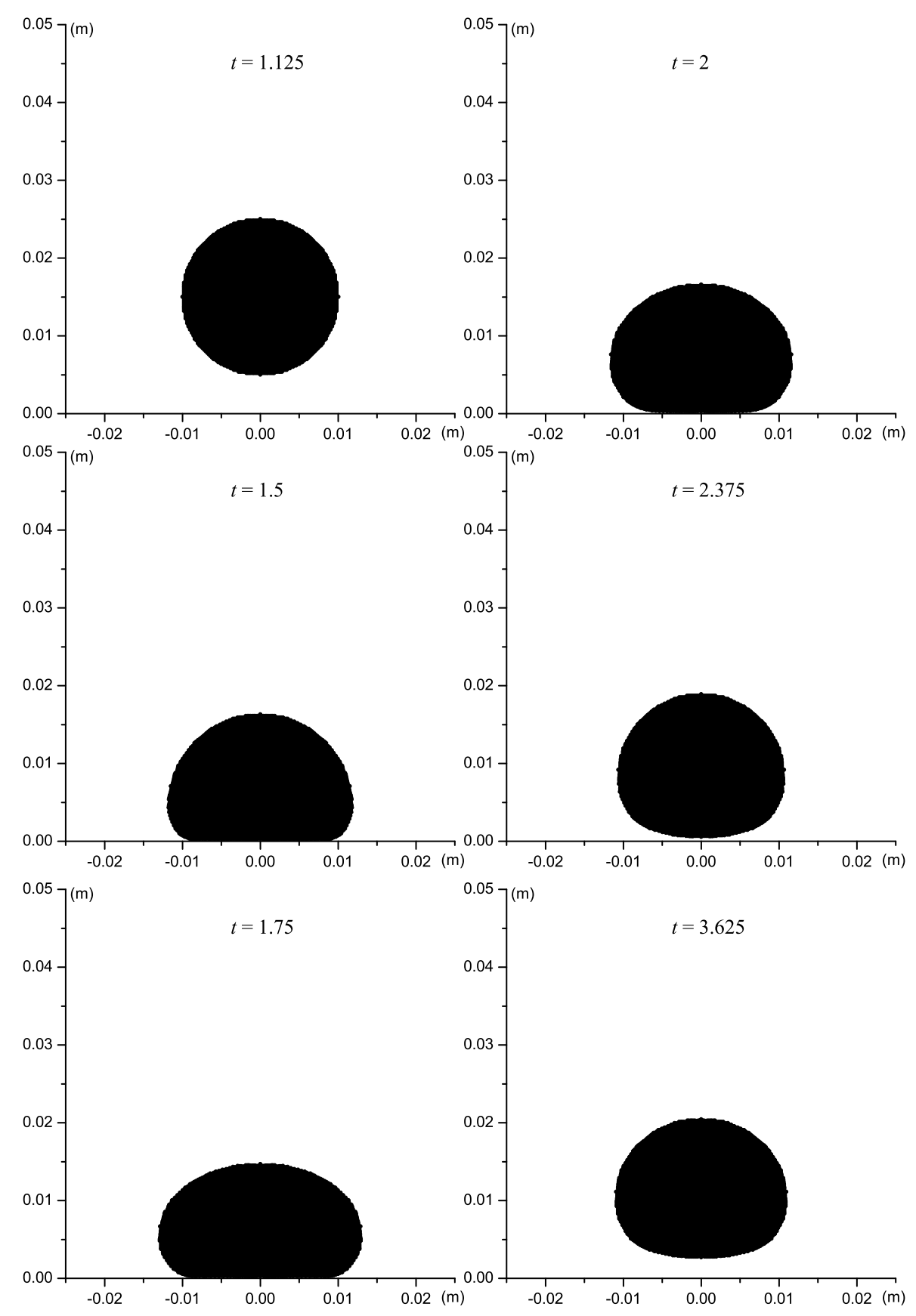

Fig. 9. SPH simulation of a falling drop of an Oldroyd-B fluid with $\nu=0.04 \mathrm{~m}^{2} \mathrm{~s}^{-1}$, $\lambda_{1}=0.02 \mathrm{~s}$ and $\lambda_{2}=0.002 \mathrm{~s}$. Fluid flow visualization at different dimensionless times $t$ as indicated.

to $\Delta d=0.01 \mathrm{~cm}$ ) again diverged with two smaller time steps $5 \times 10^{-6} \mathrm{~s}$ and $1 \times 10^{-6}$ s tried. 

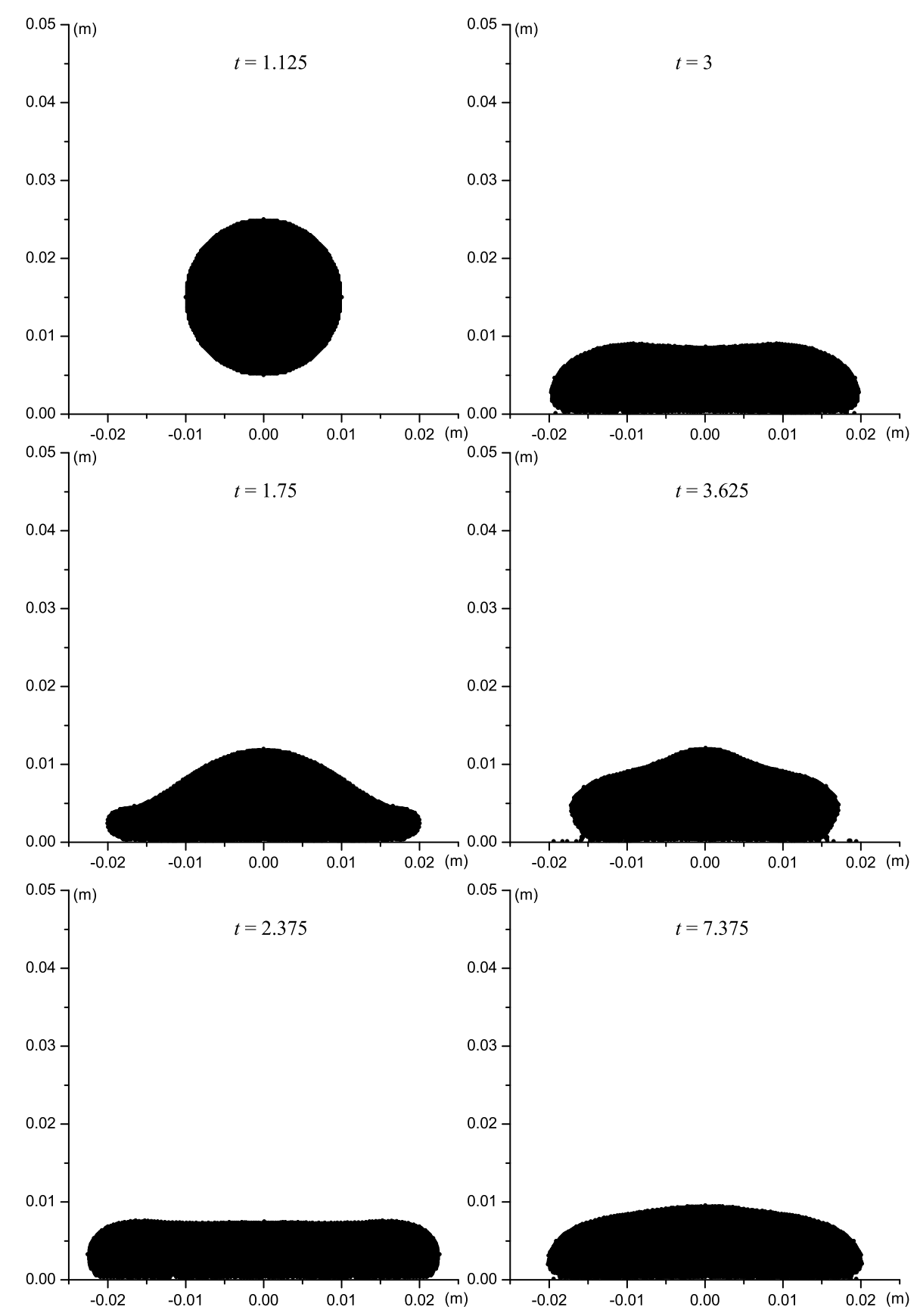

Fig. 10. SPH simulation of a falling drop of a UCM fluid with $\nu=0.004 \mathrm{~m}^{2} \mathrm{~s}^{-1}$, $\lambda_{1}=0.02 \mathrm{~s}$ and $\lambda_{2}=0 \mathrm{~s}$. Fluid flow visualization at different dimensionless times $t$ as indicated.

\section{Conclusions}

In this paper we have extended and tested a meshless method, the smoothed particle hydrodynamics (SPH) method, for unsteady free surface flows of viscoelastic fluids. The free surface flow of an Oldroyd-B fluid is considered. 
Unlike internal flows in bounded domains where the original SPH method extended to viscoelastic fluids was found to be successful, the original SPH method (either with or without artificial viscosity) does not work for the impacting drop problem studied here as a typical example of free surface viscoelastic flows. The difficulty demonstrates itself in the occurrence of nonphysical fracture due to the so-called tensile instability which has already been observed in SPH applications in elastic dynamics. For the viscoelastic case studied here, it can result in complete blowup of the computations. By adding an artificial stress term into the SPH momentum equation the tensile stress instability is overcome provided that a proper value of the coefficient for the artificial stress is chosen, which, for the problem considered here, is larger than that used habitually for problems in elastic dynamics. In addition, it is found necessary in the viscoelastic case to use an artificial stress and to retain the first term of artificial viscosity for stability reasons, while for the Newtonian case the retention of the first term in the artificial viscosity is optional. Numerical results obtained in this manner are in good agreement with those predicted by the finite difference method of Tomé et al. [15]. The proposed method is able to capture the viscoelastic effects in free surface viscoelastic flows such as bouncing.

The biggest advantage the SPH method has over grid-based methods is that it avoids the heavy tasks of re-meshing and surface tracking for problems with large deformations and possessing complex free surfaces. The price one pays for this efficiency is that the SPH method may need finer discretizations (large number of particles) to achieve an accuracy comparable with that of a gridbased method. To further improve the accuracy of the SPH method for the simulation of viscoelastic free surface flows, the incompressibility of the fluid may be enforced $[26,27]$ and various corrected versions of the SPH method [49-51] may be applied and tested.

\section{References}

[1] F.H. Harlow, The particle-in-cell method for numerical solution of problems in fluid dynamics, Proc. Symp. Applied Mathematics 15 (1963) 269-288.

[2] F.H. Harlow, E. Welch, Numerical calculation of time-dependent viscous incompressible flow of fluids with free surface, Phys. Fluids 8 (1965) 2182-2188.

[3] C.W. Hirt, B.D. Nicholls, Volume of fluid (VOF) method for dynamics of free boundaries, J. Comput. Phys. 39 (1981) 201-221.

[4] S. Osher, J.A. Sethian, Fronts propagating with curvature-dependent speed: Algorithms based on Hamilton-Jacobi formulations, J. Comput. Phys. 79 (1988) $12-49$. 
[5] R.I. Tanner, A theory of die swell, J. Polym. Sci. 8 (1970) 2067-2078.

[6] M.J. Crochet, R. Keunings, Finite element analysis of die swell of a highly elastic fluid, J. Non-Newtonian Fluid Mech. 10 (1982) 339-356.

[7] R. Keunings, An algorithm for the simulation of transient viscoelastic flows with free surfaces, J. Comput. Phys. 62 (1986) 199-220.

[8] D.W. Bousfield, R. Keunings, G. Marruci, M.M. Denn, Non-linear analysis of the surface tension driven break-up of viscoelastic filaments, J. Non-Newtonian Fluid Mech. 21 (1986) 79-97.

[9] R.Keunings, D.W. Bousfield, Analysis of surface tension driven levelling in horizontal viscoelastic films, J. Non-Newtonian Fluid Mech. 22 (1987) 219-233.

[10] D.W. Bousfield, R. Keunings, M.M. Denn, Transient deformation of an inviscid inclusion in a viscoelastic extensional flow, J. Non-Newtonian Fluid Mech. 27 (1988) 205-221.

[11] M.I. Kolte, H.K. Rasmussen, O. Hassager, Transient filament stretching rheometer. Part 2. Numerical simulation, Rheol. Acta 36 (1997) 285-302.

[12] M.W. Yao, G.H. McKinley, Numerical simulation of extensional deformations of viscoelastic liquid bridges in filament stretching devices, J. Non-Newtonian Fluid Mech. 74 (1998) 47-88.

[13] J. Cormenzana, A. Ledda, M. Laso, B. Debbaut, Calculation of free surface flows using CONNFESSITT, J. Rheol. 45 (2001) 237-258.

[14] M. Laso, H. C. Öttinger, Calculation of viscoelastic flow using molecular models: the CONNFESSITT approach, J. Non-Newtonian Fluid Mech. 47 (1993) 1-20.

[15] M.F. Tomé, N. Mangiavacchi, J.A. Cuminato, A. Castelo, S. McKee, A finite difference technique for simulating unsteady viscoelastic free surface flows, J. Non-Newtonian Fluid Mech. 106 (2002) 61-106.

[16] L.B. Lucy, A numerical approach to the testing of the fission hypothesis, Astron. J. 83 (1977) 1013-1024.

[17] R.A. Gingold, J.J. Monaghan, Smoothed particle hydrodynamics theory and application to non-spherical stars, Mon. Not. R. Astron. Soc. 181 (1977) 375389.

[18] L.D. Libersky, A.G. Petschek, Smooth particle hydrodynamics with strength of materials, in: H.E. Trease, M.J. Fritts, W.P. Crowley (Eds.), Advances in the Free-Lagrange Method, Moran, WY, USA, June 1990, vol. 395, Springer-Verlag, New York/Berlin, 1991, p. 248-257 (Lect. Notes in Phys.).

[19] L.D. Libersky, A.G. Petschek, T.C. Carney, J.R. Hipp, F.A. Allahdadi, High strain Lagrangian hydrodynamics, J. Comput. Phys. 109 (1993) 67-75.

[20] W. Benz, E. Asphaug, Simulations of brittle solids using smoothed particle hydrodynamics, Comput. Phys. Commun. 87 (1995) 253-265. 
[21] J.J. Monaghan, Simulating free surface flows with SPH, J. Comput. Phys. 110 (1994) 399-406.

[22] H. Takeda, S.M. Miyama, M. Sekiya, Numerical simulation of viscous flow by smoothed particle hydrodynamics, Prog. Theor. Phys. 92 (5) (1994) 939-959.

[23] S.J. Watkins, A.S. Bhattal, N. Francis, J.A. Turner, A.P. Whitworth, A new prescription for viscosity in smoothed particle hydrodynamics, Astron. Astrophys. Suppl. Ser. 119 (1996) 177-187.

[24] J.P. Morris, P.J. Fox, Y. Zhu, Modeling low Reynolds number incompressible flows using SPH, J. Comput. Phys. 136 (1997) 214-226.

[25] C. Maveyraud, W. Benz, A. Sornette and D. Sornette, Solid friction at high sliding velocities: an explicit three-dimensional dynamical smoothed particle hydrodynamics approach, J. Geophys. Res. 104 (1999) 28769-28788.

[26] S.J. Cummins, M. Rudman, An SPH Projection Method, J. Comput. Phys. 152 (1999) 584-607.

[27] S. Shao, E.Y.M. Lo, Incompressible SPH method for simulating Newtonian and non-Newtonian flows with a free surface, Advances Water Resour. 26 (7) (2003) 787-800.

[28] P.W. Cleary, J.J. Monaghan, Conduction modeling using smoothed particle hydrodynamics, J. Comput. Phys. 148 (1999) 227-264.

[29] J.J. Monaghan, A. Kocharyan, SPH simulation of multi-phase flow, Comput. Phys. Commun. 87 (1995) 225-235.

[30] J.P. Morris, Simulating surface tension with smoothed particle hydrodynamics, Int. J. Numer. Meth. Fluids 33 (2000) 333-353.

[31] L. Oger, S.B. Savage, Smoothed particle hydrodynamics for cohesive grains, Comput. Meth. Appl. Mech. Engrg. 180 (1999) 169-183.

[32] P.W. Cleary, M. Prakash, Discrete-element modelling and smoothed particle hydrodynamics: potential in the environmental sciences, Phil. Trans. R. Soc. Lond. A 362 (2004) 2003-2030.

[33] R. Ata, A. Soulaïmani, A stabilized SPH method for inviscid shallow water flows, Int. J. Numer. Meth. Fluids 47 (2005) 139-159.

[34] J.J. Monaghan, SPH compressible turbulence, Mon. Not. R. Astron. Soc. 335 (2002) 843-852.

[35] M. Kröger, I. Stankovic, S. Hess, Towards multiscale modeling of metals via embedded particle computer simulation, Multiscale Model. Simul. 1 (2003) 2539.

[36] M. Ellero, M. Kröger, S. Hess, Viscoelastic flows studied by smoothed particle dynamics, J. Non-Newtonian Fluid Mech. 105 (2002) 35-51. 
[37] M. Ellero, R.I. Tanner, SPH simulations of transient viscoelastic flows at low Reynolds number, J. Non-Newtonian Fluid Mech. 132 (2005) 61-72.

[38] M. Ellero, M. Kröger, S. Hess, Multiscale dynamics modeling of viscoelastic materials containing rigid non-rotating inclusions, Multiscale Model. Simul. 5:2 (2006) in press.

[39] G.R. Liu, M.B. Liu, Smoothed Particle Hydrodynamics-A Meshfree Particle Method, World Scientific, 2003.

[40] J.J. Monaghan, Smoothed particle hydrodynamics, Rep. Prog. Phys. 68 (2005) 1703-1759.

[41] J.J. Monaghan, Smoothed particle hydrodynamics, Annu. Rev. Astron. Astrophys. 30 (1992) 543-574.

[42] O. Flebbe, S. Munzel, H. Herold, H. Riffert, H. Ruder, Smoothed particle hydrodynamics-physical viscosity and the simulation of accretion disks, Astrophys. J. 431 (1994) 754-760.

[43] J.W. Swegle, D.L. Hicks, S.W. Attaway, Smoothed particle hydrodynamics stability analysis, J. Comput. Phys. 116 (1995) 123-134.

[44] J.J. Monaghan, SPH without a tensile instability, J. Comput. Phys. 159 (2000) 290-311.

[45] J.P. Gray, J.J. Monaghan, R.P. Swift, SPH elastic dynamics, Comput. Methods Appl. Mech. Engrg. 190 (2001) 6641-6662.

[46] P.M. Campbell, Some new algorithms for boundary values problems in smoothed particle hydrodynamics, DNA Report, DNA-88-286 (1989).

[47] P.W. Randles, L.D. Libersky, Smoothed particle hydrodynamics: Some recent improvements and applications, Comput. Methods Appl. Mech. Engrg. 139 (1996) 375-408.

[48] R.W. Hockney, J.W. Eastwood, Computer Simulation Using Particles, McGraw-Hill, New York, 1981.

[49] J. Bonet, T.-S.L. Lok, Variational and momentum preservation aspects of smoothed particle hydrodynamic formulations, Comput. Methods Appl. Mech. Engrg. 180 (1999) 97-115.

[50] J.K. Chen, J.E. Beraun, A generalized smoothed particle hydrodynamics method for nonlinear dynamic problems, Comput. Methods Appl. Mech. Engrg. 190 (2000) 225-239.

[51] M.B. Liu, W.P. Xie, G.R. Liu, Modeling incompressible flows using a finite particle method, Appl. Math. Model. 29 (2005) 1252-1270. 